\title{
通过串联反应不对称合成氧化吲哚螺环化合物的研究进展
}

\author{
肖永龙 ${ }^{a}$ 周宇 ${ }^{b}$ 王 江 ${ }^{b}$ 王进欣*,a 柳 红*,b \\ ( ${ }^{a}$ 中国药科大学江苏省药物分子设计与成药性优化重点实验室 南京 210009) \\ ${ }^{b}$ 中国科学院上海药物研究所受体结构与功能重点实验室 上海 201302)
}

\begin{abstract}
摘要 氧化吲哚螺环类结构因具广泛的生物活性, 已逐渐成为一类重要的类药性骨架. 因此, 开发简单高效的合成方 法构建复杂的氧化吗哚螺环类化合物库已经成为化学工作者广泛关注的研究方向之一. 由于现代有机化学倍受中间产 物分离提纯及官能团保护、脱保护策略等的困扰, 而串联反应的出现为克服以上诸多难题提供了机遇, 且在合成具有 光学活性的天然产物和复杂分子中显示了它潜在的优势. 近年来, 通过有机串联反应策略不对称合成氧化吲哚螺环类 化合物备受关注, 大量的研究工作被报道. 分别从 $C(3)$ 位不饱和氧化吲哚衍生物、饱和氧化弨哚衍生物、 $C(3)$ 位无取代 氧化吲咏衍生物以及非氧化吲哚衍生物四大类起始原料出发, 简单综述了近 5 年来氧化吲槑螺环化合物的合成方法进 展, 并对各类反应对底物要求、反应条件、反应选择性、产率以及机理的研究进行了讨论和总结.
\end{abstract}

关键词＼cjkstart氧化吲哚螺环化合物; 不对称合成; 催化剂; 串联反应

\section{Asymmetric Synthesis of Spirooxindoles via Organocascade Strategies}

\author{
Xiao, Yonglong ${ }^{a} \quad$ Zhou, Yu $^{b} \quad$ Wang, Jiang $^{b} \quad$ Wang, Jinxin $^{*, a} \quad$ Liu, Hong ${ }^{*, b}$ \\ ( ${ }^{a}$ Jiangsu Province Key Laboratory of Drug Design and Optimization Medicine, China Pharmaceutical University, \\ Nanjing 210009) \\ ( ${ }^{b}$ CAS Key Laboratory of Receptor Research, Shanghai Institute of Materia Medica, Chinese Academy of Sciences, \\ Shanghai 201203)
}

\begin{abstract}
The spirooxindoles have broad and promising activities in various therapeutic areas, and become a privileged skeleton for drug discovery. Therefore, the development of some simple and efficient strategies to build these sophisticated scaffolds has become one of the most widespread concerns. However, the traditional methods are limited by the separation and purification of intermediates, the functional group protection and de-protection. Recently, the cascade strategies have shown special advantages in the synthesis of optically active natural products and complex molecules, many related studies have been reported. This review summarizes the enantioselective synthesis of spirooxindoles via cascade strategies in the past five years and organized on the basis of four types of starting materials: unsaturated oxindole derivatives, $\mathrm{C}(3)$-substituted oxindoles, $\mathrm{C}(3)$-unsubstituted oxindoles and nonoxindoles.
\end{abstract}

Keywords spirooxindoles; asymmetric; catalysis; organocascade

氧化吲哚螺环是一类重要的类药性骨架, 广泛存在 于天然产物和生物活性分子中. 如图 1 所示, 钩吻碱 (Gelsemine) 是一类常见的生物碱, 具有广泛的生物学活 性 ${ }^{[1]}$; Spirotryprostatins A 和 B 在哺乳动物 tsFT210 细胞 中表现出良好的抗 $\mathrm{G} 2 / \mathrm{M}$ 细胞转移活性 ${ }^{[2]}$. 另外一些合 成的螺环骨架化合物, 如 MI-219 能够有效抑制肿瘤细 胞的 p53 和 E3 泛素连接酶 MDM2 之间的相互作用 ${ }^{[3]}$;
NITD609 具有较好的抗疮作用; Notoamide A 表现出显 著的细胞毒素活性; Strychnofoline 能有效地抑制抗真核 细胞的有丝分裂; Rhynchophylline 是 $N$-甲基- $D$-天冬氨 酸受体拮抗剂; $(S)$-Spirobrassinin 是一种天然的植物抗 毒素等. 鉴于氧化吲哚螺环化合物广泛的生物活性和其 潜在的治疗用途 ${ }^{[4]}$, 因此, 发展新的高效的合成方法用 于构建手性螺环氧化吲哚类化合物具有重要的意义.

\footnotetext{
*E-mail: hliu@mail.shcnc.ac.cn, jinxinwang@163.com

Received April 28, 2015; revised June 16, 2015; published online July 2, 2015.

Project supported by the National Natural Science Foundation of China (No. 21372235).

国家自然科学基金(No. 21372235)资助项目.
} 


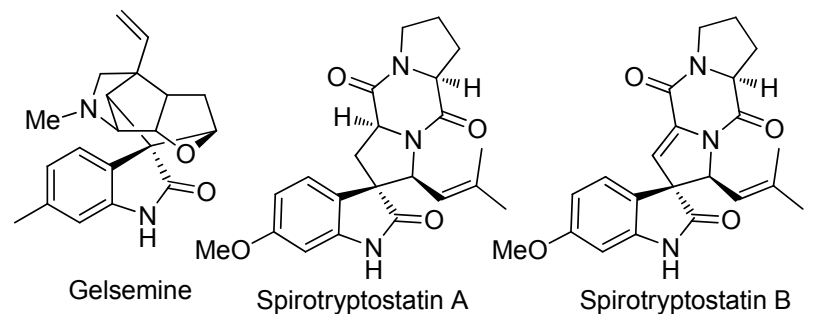<smiles>CC(C)(C)CC1N[C@H](C(=O)NCC[C@H](O)CO)[C@@H](c2cccc(Cl)c2)[C@@]12C(=O)Nc1cc(F)c(Cl)cc12</smiles>

MI-219

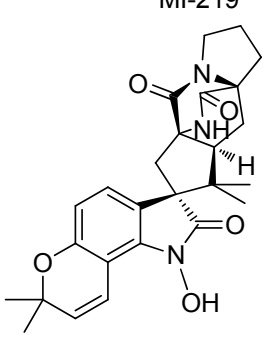<smiles>CC[C@H]1CC2CC[C@@]3(C(=O)Nc4ccccc43)C2C[C@H]1/C(=C\OC)C(=O)O[Na]</smiles>

Rhynchophylline

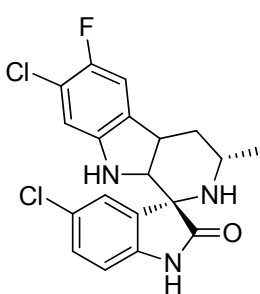

NITD609

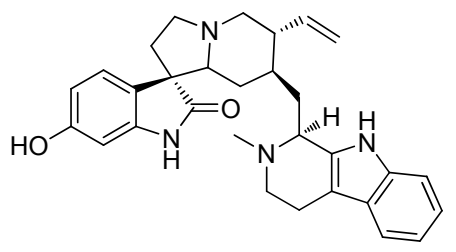

Strychnofoline<smiles>CSC1=NCC2(S1)C(=O)Nc1ccccc12</smiles>

(S)-Spirobrassinin

图 1 具有氧化吲哚螺环的天然产物或药物分子

Figure 1 Natural products and pharmaceutical molecules containing a spirooxindole core structure

但是, 如何设计简便易操作的方法来合成这些复杂 的螺环分子却成为有机化学研究领域的巨大挑战. 例如 当吲哚 $\mathrm{C}(3)$ 位被双键取代时, 由于双键两端的碳原子受 取代基的影响较大，因此很难获得好的区域选择性; 另 外氧化吲哚螺环产物的最大特点在于 $\mathrm{C}(3)$ 位碳原子上 有一个不对称的环，环上的不同取代基可能会导致产物 中更多手性中心的形成, 这要比催化剂诱导的手性中心 形成更加具有挑战性; 另外, 很多反应的确切反应机理 尚未得到完全阐明.

在过去的几年里, 新颖高效的串联反应策略在构建 复杂螺环分子中起到了非常重要的作用 ${ }^{[5]}$. 本文对近五 年来通过串联反应策略合成复杂的手性吲哚螺环的方 法进行了简单的综述. 如图 2 所示, 根据起始原料的不 同主要分为以下四类进行论述: (1) 以 $\mathrm{C}(3)$ 位不饱和氧化 吲哚衍生物为起始原料, 例如甲基氧化吲哚、滇红以及 isatinimine, 这些不饱和化合物与设计的具有亲电或者 亲核位点的化合物通过环加成反应形成吲哚螺环环合 物; (2) 以 $C(3)$ 位取代的饱和氧化吲哚衍生物为起始原

料; (3)以 C(3)位无取代氧化吲哚衍生物为起始原料, 这 类化合物可以与另外两个反应物或者同时具有两个亲 电中心的一个反应物发生反应; (4)非氧化吲哚衍生物为 起始原料，如芳香酫等.

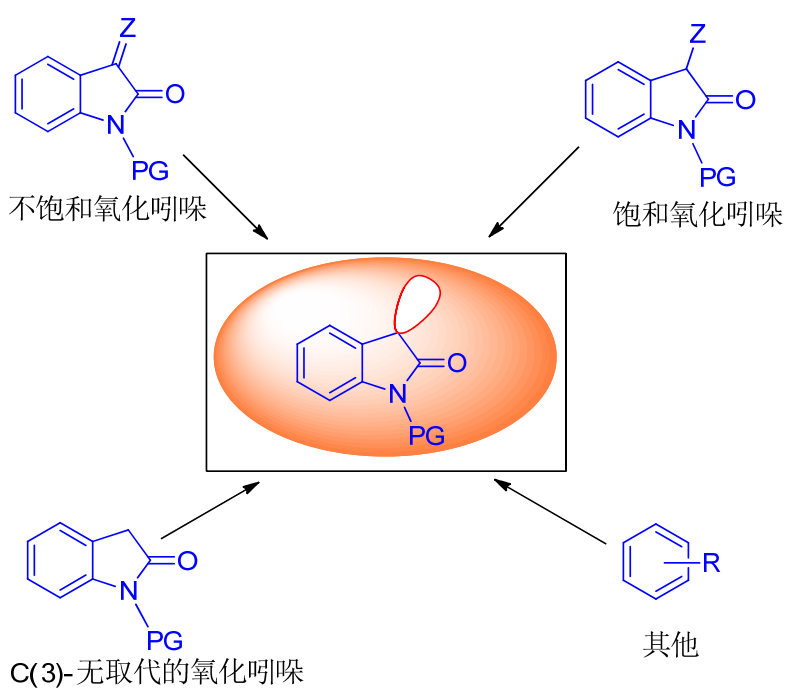

图 2 四类不同起始原料合成氧化吲哚螺环骨架

Figure 2 Four different types of starting materials to synthesize spirooxindole core structure

\section{1 吲哚螺环化合物的合成}

\section{$1.1 \mathrm{C}(3)$ 位不饱和氧化吲哚衍生物为起始原料}

1.1.1 $\mathrm{C}(3)$ 位 $\mathrm{C}=\mathrm{C}$ 不饱和氧化吲哚作为亲电/亲核试 剂

不同结构类型的不饱和氧化吲哚衍生物通过与不 同的底物在不同催化剂的作用下，可以制备结构多样化 且具有一个或多个手性中心的吲哚螺环类化合物. 在 $\mathrm{C}(3)$ 位 $\mathrm{C}=\mathrm{C}$ 不饱和氧化吲哚衍生物参与的反应中, 手 性一级胺和二级胺类催化剂被广泛地应用, 主要由于这 类催化剂能够很好地与底物分子形成烯胺或亚胺类中 间体，从而促进其与亲核试剂和亲电试剂的连续加成. 在早期的报道中，吡咯烷以及吡咯烷醚类催化剂被广泛 应用 ${ }^{[6]}$, 但近年来 Hayashi-Jørgensen 类吡咯烷催化剂在 构建手性螺环化合物中表现出更好的催化活性.

2009 年, Melchiorre 等 ${ }^{[7]}$ 报道了一个 HayashiJørgensen 类吡咯烷催化剂催化的三级 Michael/Michael 分子间的级联消除反应. 在该反应过程中，氧化吲哚衍 生物 1、脂肪醛 2 以及 $\alpha, \beta$-不饱和醛 3 在 HayashiJørgensen 类吡咯烷催化剂的催化下形成吲哚螺环己烷 类产物 4 , 反应产率良好且具有较高的非对映和对映选 择性(Scheme 1). 另外, Chen 课题组 ${ }^{[8]}$ 也在 HayashiJørgensen 类催化剂的作用下成功实现了类似的螺环化 合物 6 的构建，获得 99：1 的非对映选择性和 99\%的对 
映选择性(Scheme 1).

2011 年, Melchiorre 等 ${ }^{[9]}$ 首次实现了手性胺催化剂 C-1 催化 3-烯基氧化吲哚 7 与烯基取代的 $\alpha, \beta$-不饱和醛 8 之间的不对称 Diels-Alder 反应. 该反应不仅具有较好 的收率和较高的对映选择性, 最重要的意义在于该反应 可以把底物 8 由吡咯环拓展成吡定环或呋喃环, 从而大 大增加了该反应在药物化学领域的应用 (Scheme 2).
2013 年, Feng 等 ${ }^{[10]}$ 首次利用手性 $\mathrm{Mg}\left(\mathrm{ClO}_{4}\right)_{2}$ 与 $N, N^{\prime}$-双氧 化物配体复合物为催化剂, 以 3-烯基氧化吲哚 10 和氯 取代氰亚胺类化合物 11 为起始原料, 一锅快速构建吲 哚螺吡唑啉类衍生物 12, 该方法不仅具有较高的收率 和较好的立体选择性，而且反应条件温和、操作简便 (Scheme 2). 2014 年, Wang 等 ${ }^{[11]}$ 发现以 $\alpha, \beta$-不饱和醛 14<smiles>[R]C=c1c2c[X]:[R]ccc=2n([TlH])c1=O</smiles>

1<smiles>[R]C=C1C(=O)N(C(=O)OCC2=CC#[R11]=CC=C2)c2cc[R1]#cc21</smiles>

5<smiles>[R]CC=[O+]</smiles><smiles>[R4]C=CC=O</smiles>

3
$15 \%$ C-1 $15 \%$ OFBA toluene, $40^{\circ} \mathrm{C}$ $35 \% \sim 74 \%$ yield up to $>19: 1 d r$ $>99 \%$ ee

$15 \%$ C-1

$15 \% \mathrm{BA}$

$\underset{50 \% \sim 88 \% \text { yield }}{\stackrel{\mathrm{CH}_{3} \mathrm{CN} \text {, r.t. }}{\longrightarrow}}$

up to $>99: 1 d r$ $88 \% \sim>99 \%$ ee

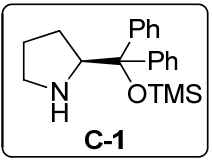<smiles>[R]C1C=C(C)C([R])C2(C(=O)N([2H])c3cc[R1]([H])cc32)C1[R]</smiles>

$\mathrm{OHC}$<smiles>[R]C1C=C(CC)C([R])C2(C(=O)N(C([R])([R])C)c3cc[R1]cc32)C1[R]</smiles>

图式 1 氧化吲哚衍生物、醛以及 $\alpha, \beta$-不饱和醛之间的级联反应

Scheme 1 Cascade reaction of methyleneoxindoles, aliphatic aldehydes and $\alpha, \beta$-unsaturated aldehydes<smiles>[R]C=C1C(=O)N([Pb])c2cc[R1]#cc21</smiles>

7<smiles>[R]C=C1C(=O)N(C(C)(C)C)c2cc[R1]#cc21</smiles>

10<smiles>[Z]C=Cc1c(C)n(C(=O)OCc2ccccc2)c2ccc([R])cc12</smiles>

8

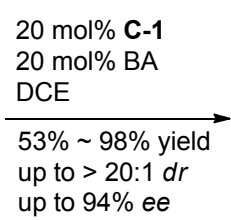

up to $94 \%$ ee

$10 \%$ C-2, DIPEA $\mathrm{DCE}, 60^{\circ} \mathrm{C}$ up to $98 \%$ yield up to $99 \%$ ee

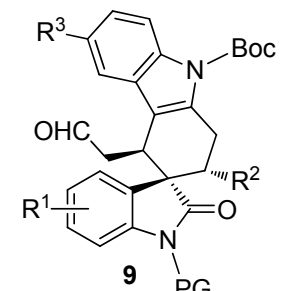

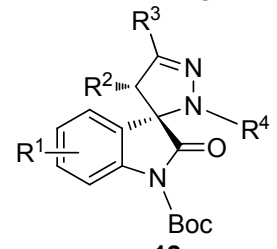

11 12

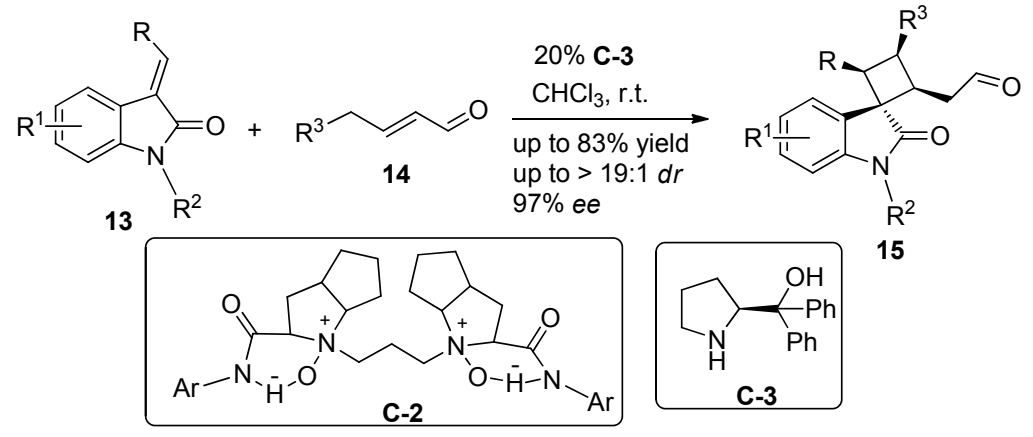

图式 2 以 3-烯基-氧化吲哚为起始原料反应构建多手性中心吲哚螺环骨架

Scheme 2 3-Enyl-oxindole as starting material to build more chiral centers spirooxindoles 
与 3-烯基氧化吲哚 13 为起始原料反应, 通过 $[2+2]$ 环加 成反应, 能够构建吲哚螺环丁烷骨架的化合物 $\mathbf{1 5}$, 该反 应不仅具有较高的收率以及较好的立体选择性, 而且一 锅构建四个连续手性中心(Scheme 2).

小分子可以通过与底物分子中的氮原子或氧原子 上的孤对电子形成氢键来增强底物分子的亲电性. 化学 家们据此设计出了一类新型的氢键类催化剂, 设想通过 催化剂分子与底物形成氢键来诱导反应的发生以及立 体选择性的控制 ${ }^{[12]}$. 在此类氢键催化剂中, 硫脲类双功 能催化剂展现出了高效的催化活性 ${ }^{[13]}$.

2011 年, Barbas 等 ${ }^{[14]}$ 发现以两分子结构不同的吲哚 烯 16 和 17 为起始原料, 在双功能硫嫝催化剂 C-4 的催 化下，通过 Diels-Alder 反应可以高效地构建吲哚螺咔唑 类化合物 18. 该反应不仅可以以较高的产率和立体选 择性获得一系列具有该骨架结构的氧化吲哚螺环产物, 溶剂和催化剂的可循环利用更是该反应的一大优点 (Scheme 3). 2013 年, Zhu 等 ${ }^{[15]}$ 引入硝基烯的底物 19, 采 用类似的方法, 一锅串联构建了类似的氧化吲哚螺环骨 架 20; 在该反应中, 通过手性叔胺类催化剂的催化, 两 个底物分子经 oxa-和 aza-Michael-Michael 一锅串联反 应，高效合成了相应的螺环化合物，不仅具有原料廉价 易得、反应条件温和的优点，同时还获得了较好的收率，

较高的对映选择性和非对映选择性(Scheme 3).

后来, Barbas 等 ${ }^{[16]}$ 在手性磷化氢催化剂作用下，率 先实现了不饱和氧化吲哚 21 与烯丙基酯衍生物 22 的 $[3+2]$ 环加成反应，该反应不仅产率高立体选择性好， 而且一锅获得了具有多个手性中心的吲哚螺环戊烷结 构骨架 23. 由于该过程操作简单，反应条件温和以及产 物结构的多样性, 已被广泛地应用于药物化学领域 (Scheme 3). 2013 年, Wang 等 ${ }^{[17]}$ 采用手性硫艮催化剂 C-6, 以鲐内酯 24 和吲哚烯 21 作为起始原料，高收率高 选择性地实现了二氢吡咯类吲哚螺环 25 的合成. 他们 首次实现了利用唤酯 $\mathrm{C}(4)$ 位的亲核性以及 $\mathrm{C}(2)$ 位的 亲电性来构建吲哚螺环(Scheme 3).

亲核类催化剂因其良好的灵活性、动态性而深受合 成化学家的青睐, 如具有电子对供体的手性磷化氢催化 剂就是一类非常有效的催化剂 ${ }^{[18]}$. 2010 年, Marinetti 等 ${ }^{[19]}$ 在 BINOL 衍生化的磷化氢催化剂的作用下, 实现 了底物 26 和联烯酯 27 之间的 $[3+2]$ 环加成反应，构建 了 “ $\gamma$-加合” 的吲哚螺环戊烷类产物 28, 且该反应具有 较高的产率以及较好的立体选择性. 后来，他们又以类 似的底物, 实现了其他两类吲哚螺环骨架 30 和 33 的构 建，也获得较好的收率和立体选择性(Scheme 4)。

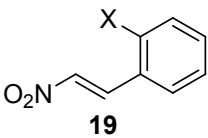

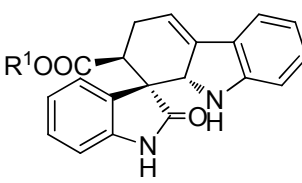

18

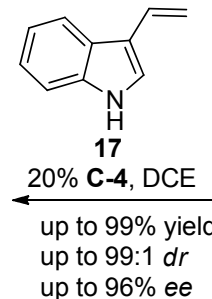

up to $96 \%$ ee

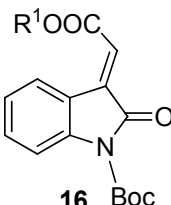

16 Boc

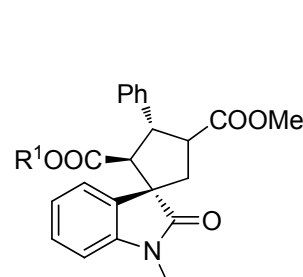

$23 R$

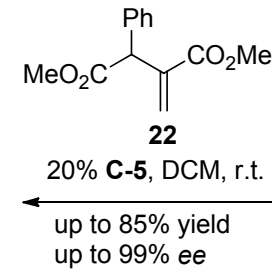

21

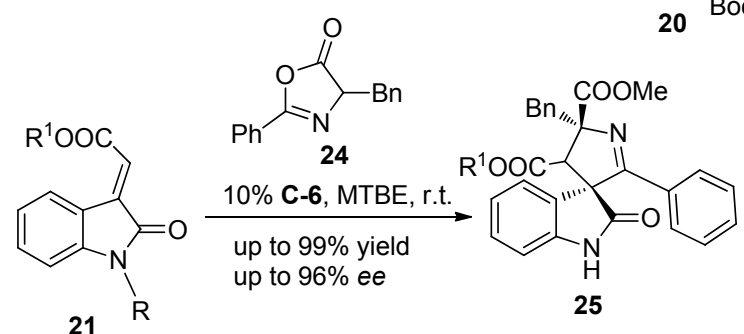

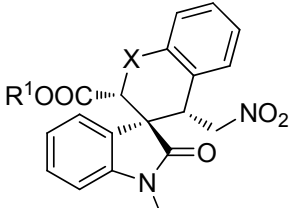

20 Boc
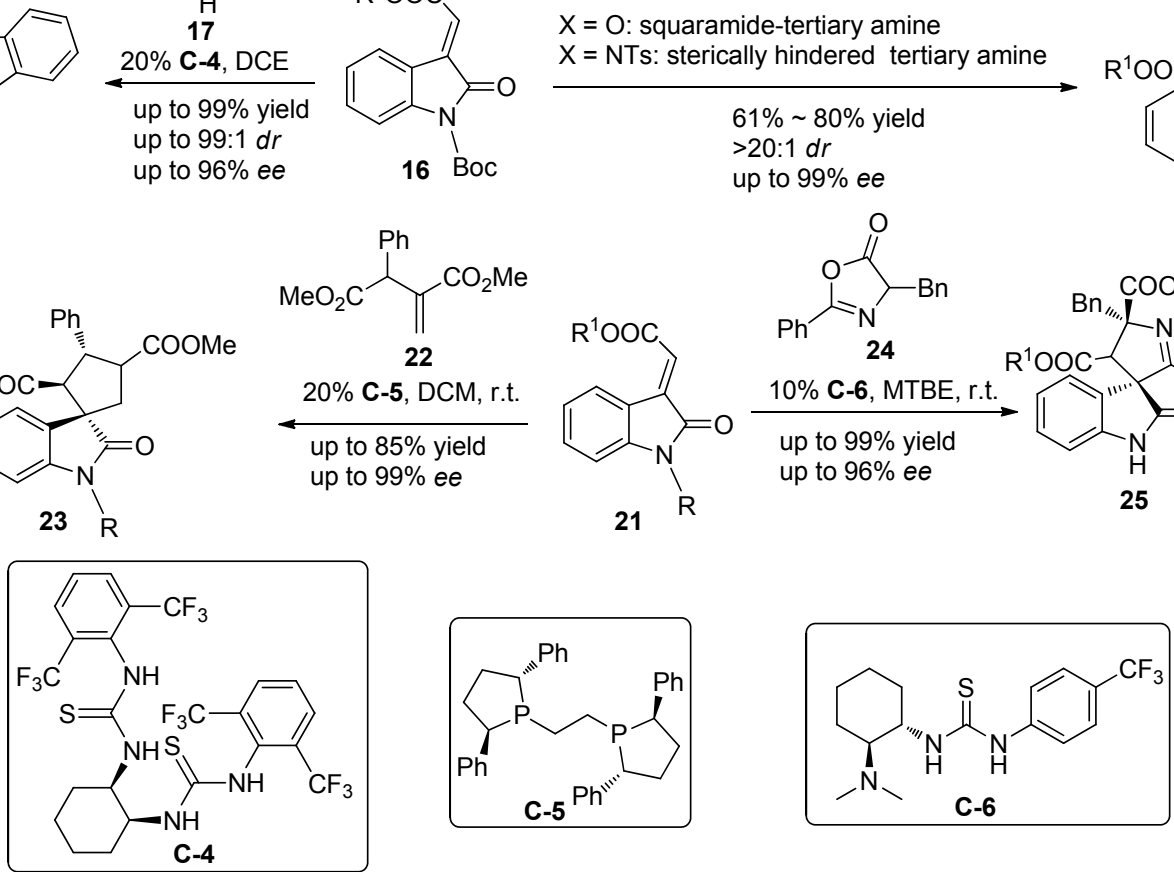

图式 3 吲哚烯与不同类型的底物反应构建不同的吲哚螺环骨架

Scheme 3 Reactions of indole-ene with different substrates to construct spiroindoles 


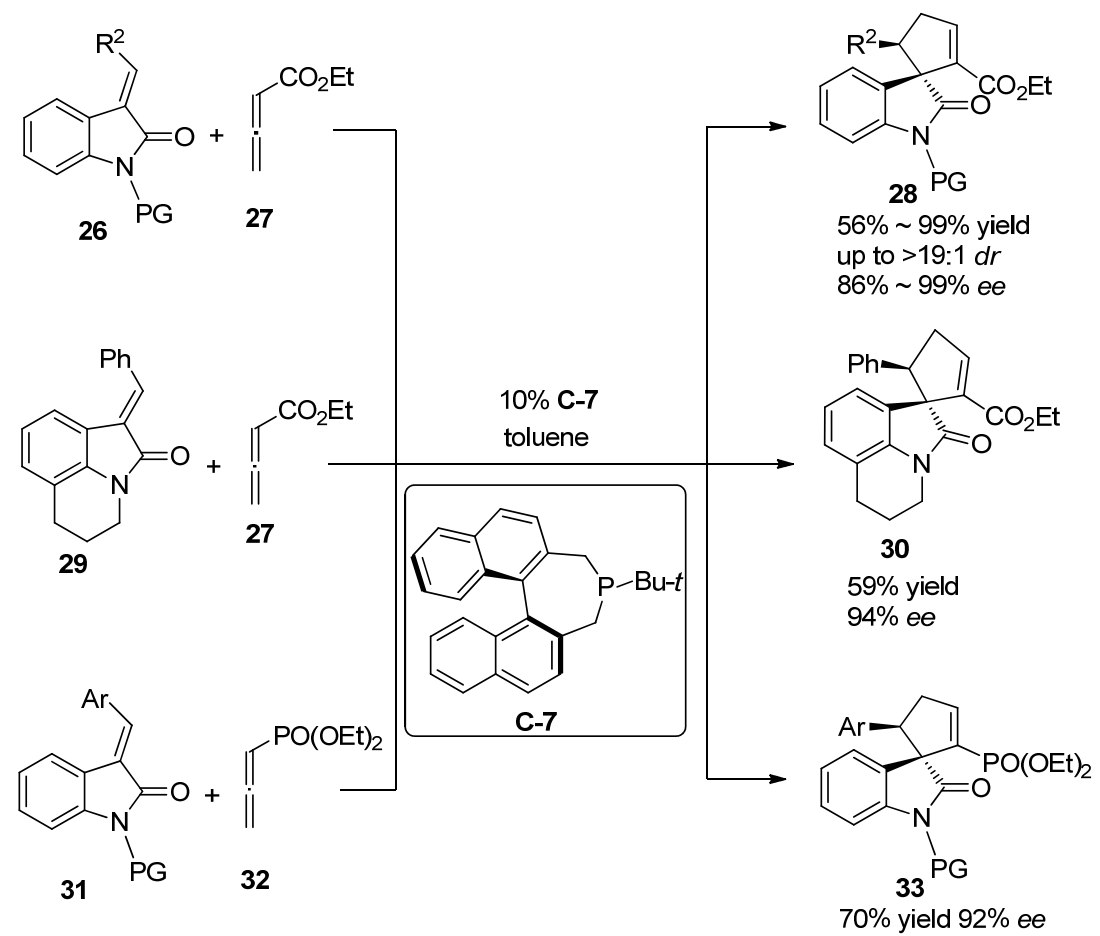

图式 4 手性磷酸催化吲哚烯与联烯之间的[3+2]环加成反应

Scheme 4 Chiral phosphine-catalyzed [3+2] cyclization between methyleneoxindoles and allenes

近年来，手性磷酸及其衍生物作为一种布朗斯特酸 催化剂和双功能催化剂已经得到了广泛的应用.

2009 年, Gong 等 ${ }^{[20]}$ 首次报道了一种吲哚螺吡咯环 骨架 36 的高效、高选择性的合成方法. 他们研究发现在 手性磷酸催化剂的催化下, 通过 N-Ac 保护的吲哚烯、 醛以及氨基酯之间的反应不但可以获得高的对映选择 性，而且还实现 “反常” 的区域化学选择性，制备了四 氢吡咯类吲哚螺环结构. 这种直接高效高选择性构建吲 哚螺环骨架的方法给药物化学家合成功能性骨架提供 了依据. 通过理论计算, 他们发现该反应的高选择性主 要是由于反应过程中产生的偶氮甲碱和氧化吲哚衍生 物与磷酸催化剂形成氢键, 其反常的区域化学选择性可 能主要是由于氧化吲哚与共轭酯之间的 $\pi-\pi$ 相互作用所 致(Scheme 5). 2013 年, Antilla 等 ${ }^{[21]}$ 也通过手性磷酸镁盐 类催化剂, 以二烯醇醚 38 为起始原料, 与 $\mathrm{C}(3)$ 不饱和氧 化吲哚 37 通过 Diels-Alder 反应快速构建 C(3)位全碳取 代的具有六元环结构的氧化吲哚螺环骨架 39. 该过程 不仅条件温和、绿色环保, 最重要的是通过该方法合成 的吲哚螺环骨架是天然产物钩吻碱的核心骨架 (Scheme 5).

2011 年, $\mathrm{Lu}$ 组 ${ }^{[22]}$ 实现了滇红衍生的四元取代联烯 40 与 $\mathrm{MBH}$ 碳酸盐 41 之间的 [3+2]环加成反应构建具有 重要生物学功能的吲哚螺环戊烯氧化吲哚产物 42, 该
过程是在苏氨酸衍生的硫脲催化剂 $\mathbf{C - 1 0}$ 的催化下进行 (Scheme 6). 2012 年，他们 ${ }^{[23]}$ 首先实现了手性硫脲类催 化剂 C-11 催化的类似的滇红衍生物 43 与联烯 44 之间 的 $[4+2]$ 环合反应，同样的，该反应不仅具有较高的收 率、较好的对映选择性和非对映选择性, 通过该反应可 以得到具有重要生物学功能的 3-吲哚螺环己烯-2-氧化 吲哚 45 (Scheme 6).

除了上述有机催化剂外，一些无机金属盐催化剂也 参与了氧化吲哚螺环类化合物的合成. 2014 年, Thennarasu 等 ${ }^{[24]}$ 首先报道了一个利用 $\mathrm{Cu}$ 盐催化 $\mathrm{C}(3)$ 位 $\alpha, \beta$ 不饱和酩取代的氧化吲哚 46 与叠氮类化合物 47 一锅构 建 3,3'-双吲哚螺吡咯烷类化合物 48 的反应，该反应的 重要意义在于: (1) 一步构建两个 $\mathrm{C}-\mathrm{C}$ 键; (2) 以较高的 收率得到具有四个连续手性中心的螺环产物, 其中两个 手性为四取代的手性中心; (3)以高区域选择性和非对映 选择性得到同分异构体中的单一产物(Scheme 7). Franz 等 ${ }^{[25]} \mathrm{C}(3)$ 使用 $\mathrm{Ti}$ 盐催化剂, 实现了 $\mathrm{C}(3)$ 位 $\alpha, \beta$-不饱和酮 取代的氧化吲哚 46 与 5-烷氧基-2-芳基噁唑 49 合成二氢 吡咯氧化吲哚螺环化合物 50, 除了具有反应条件温和 的优点外，该反应其最大的特点在于以非手性催化剂可 以高非对映选择性地制备具有多个手性中心的目标产 物(Scheme 7). 


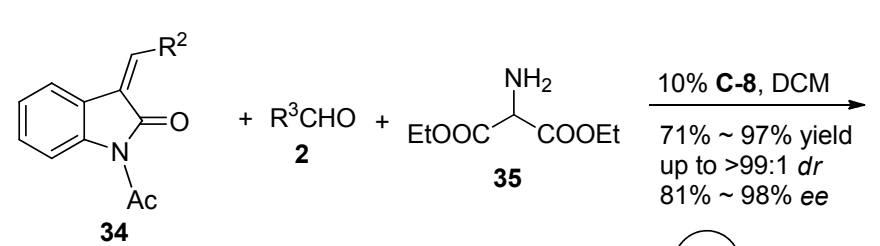<smiles>[R]C1NC(C(=O)OCC)(C(=O)OCC)C(C)(C)C12c1ccccc1N([Z4])C2[R]</smiles>

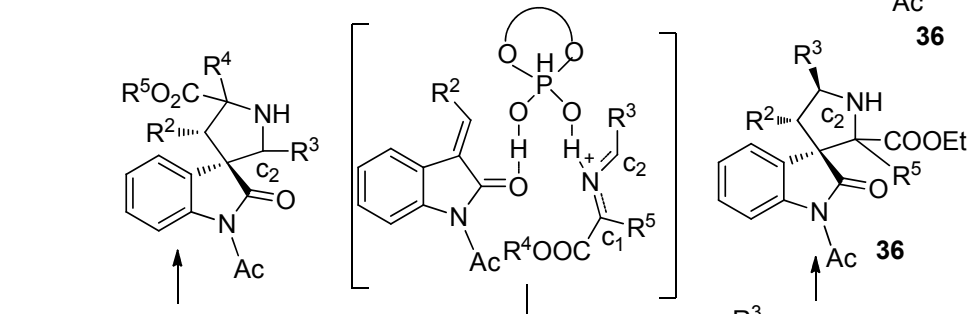

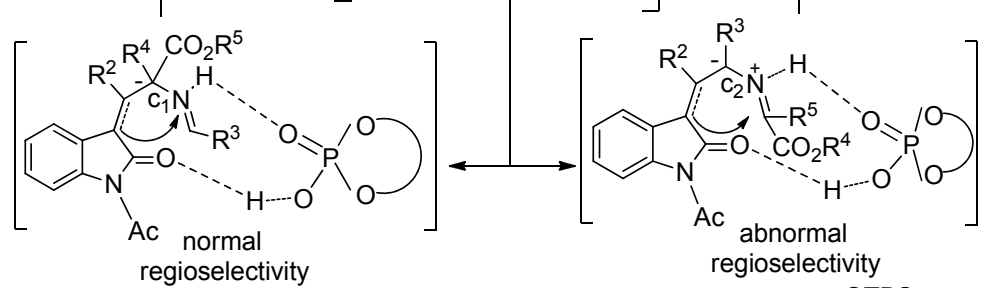

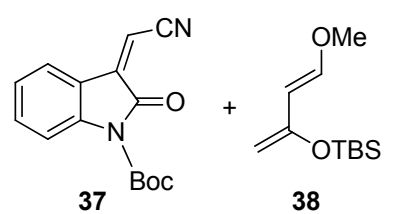

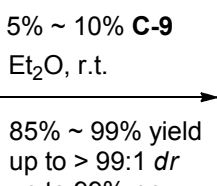
OTBS

38 up to $99 \%$ ee

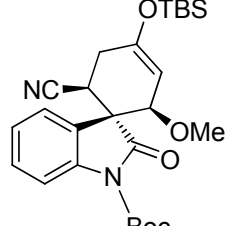<smiles>O=P1(O)Oc2cc3ccccc3cc2-c2c(c(-c3ccc4ccccc4c3)cc3ccccc23)O1</smiles>

$39^{\mathrm{Boc}}$

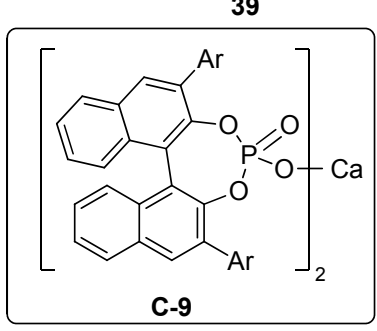

图式 5 手性磷酸盐催化构建具有生物学功能的吲哚螺环骨架

Scheme 5 Synthesis of bioactive spirooxindoles catalyzed by phosphoric acid

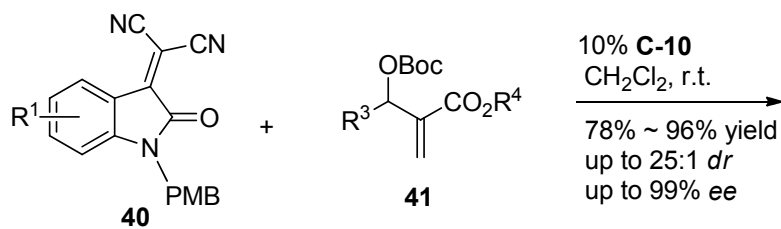

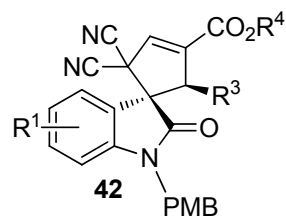<smiles>C=C(CC(=C)C(=O)OCC)OCC</smiles>

$5 \mathrm{~mol} \% \mathrm{C}-11$ toluene up to $96 \%$ yield 99:1 $d r, 93 \%$ ee
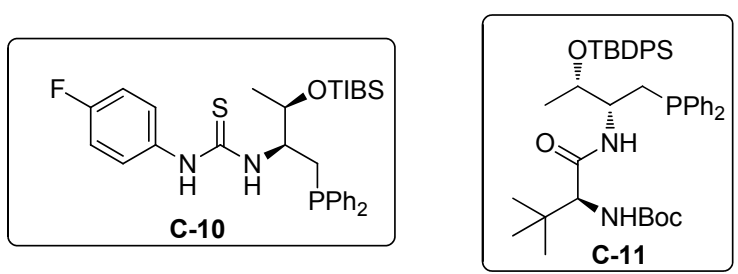

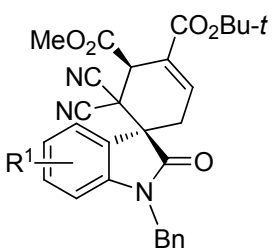

45

图式 6 滇红衍生的四元取代联烯为起始原料构建吲哚螺环骨架

Scheme 6 Using isatin-derived alkenes as starting materials to build spirooxindoles 


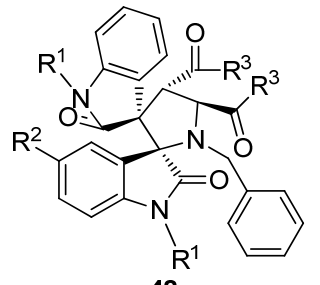

48

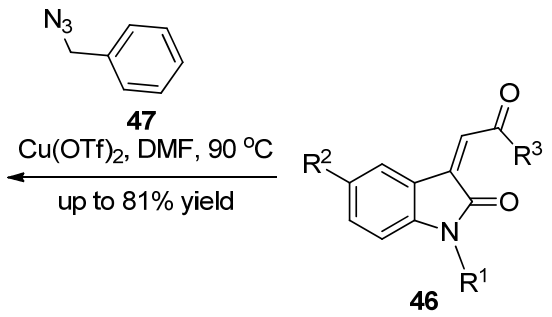

$46^{\mathrm{R}}$
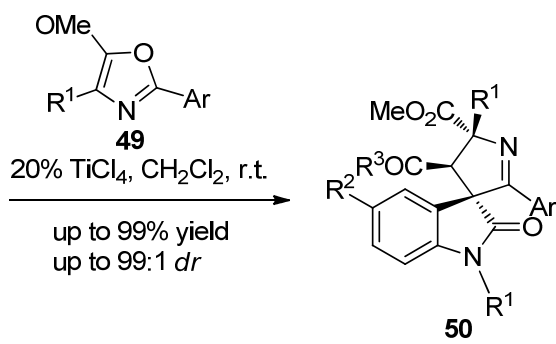

图式 7 无机金属盐催化构建吲哚螺环骨架

Scheme 7 Synthesis of spirooxindoles catalyzed by inorganic metal salts

\subsection{2 $\mathrm{C}(3)$ 位 $\mathrm{C}=\mathrm{N}$ 不饱和氧化吲哚作为亲电试剂}

含多氮的螺环骨架化合物是一类具有重要生物学 功能优势骨架, 如 NITD609 已用于候选药物的研究. 近 年来, 我们课题组 ${ }^{[26]}$ 也开展了系列的氧化吲哚螺环合 成方法学的研究. 通过研究我们发现以滇红衍生的酮亚 胺 51 和氰基取代的炔烃 52 为起始原料, 可在金鸡纳碱 类催化剂 C-12 和金复合物的共同催化下, 实现一锅 Mannich/hydroamination 串联反应, 快速构建含多氮的 氧化吲哚螺环类化合物 $\mathbf{5 3}$, 该反应获得了较好的收率 和立体选择性. 同时可用于具有生物活性的吲哚螺环骨 架的构建(Scheme 8). 手性氮杂环卡宾催化剂(chiral $N$-hetercyclic carbene catalyst)作为一类新型的有机路易 斯碱, 已被广泛地应用在有机合成中, 在构建各种杂环 化合物的策略中占有重要的位置. 2014 年, Chi 等 ${ }^{[27]}$ 采用 NHC 催化剂 C-13, 以类似的滇红衍生的酮亚胺 54 和 $\alpha, \beta$-不饱和醛 3 为起始原料, 高效实现了以 $[3+2]$ 环加成 反应制备了一类含多氮的氧化吲哚螺环类化合物 $\mathbf{5 5}$,

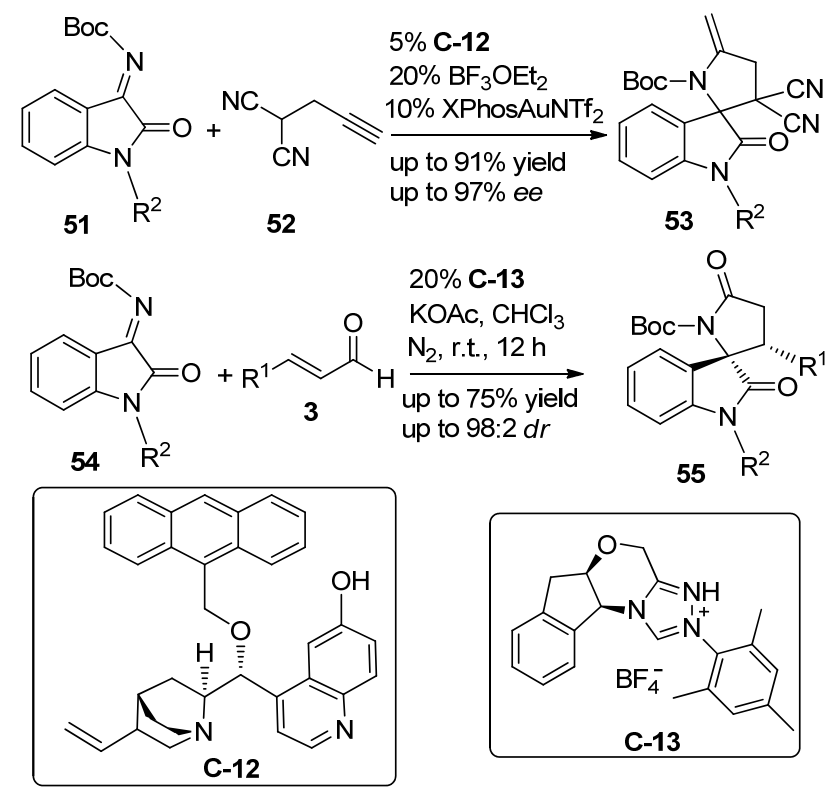

图式 8 滇红衍生的酮亚胺为起始原料构建吲哚螺环骨架 Scheme 8 Ketimine derivatived by isatin as starting materials to build spirooxindoles
该反应具有较好的收率以及较高的立体选择性(Scheme 8).

\subsection{3 $\mathrm{C}(3)$ 位 $\mathrm{C}=\mathrm{O}$ 不饱和氧化吲哚作为亲电试剂}

Bencivenni ${ }^{[28]}$ 和 Franz ${ }^{[29]}$ 课题组分别以滇红 56 和色 胺衍生物 57 为原料, 通过 Pictet-Spengler 反应巧妙地构 建了两类含多氮螺环骨架的化合物 58. 在 Bencivenni 等 的方法中, 无论选用 $R$ 型还是 $S$ 构型的磷酸催化剂, 都 能得到具有相同绝对构型的螺环产物, 这可能预示双荎 基体系的取代基对反应的立体选择性具有重要的影响 (Scheme 9). 近年来, $\alpha$-异硫氰酸衍生物作为一种亲核试 剂在不对称合成氧化吲哚螺环化合物方面得到广泛应 用. 如 2010 年 Wang 等 ${ }^{[30]}$ 以滇红衍生物 56 为起始原料, 与 $\alpha$-异硫氰酸亚胺衍生物 59 实现一锅 aldol/cyclization 串联反应. 通过催化剂篎选, 他们发现双功能的硫脲催 化剂能够以较高的收率和较好的对应选择性以及非对 应选择性合成手性的氧化吲哚螺环 61, 其反应机理可 能是 $\mathrm{N}$ 保护的缺电子的滇红被催化剂中的两个氢原子 活化, 而另外一个底物分子则被催化剂中的三级胺部分 烯醇化所致(Scheme 9).

2012 年, Scheidt 等 ${ }^{[31]}$ 报道了一类以 $\alpha, \beta$-不饱和醛 3 为起始原料, 在 $\mathrm{NHC}$ 的催化下与 $\mathrm{N}$ 取代滇红 62 实现高 效的 $[3+2]$ 环加成反应制备具有五元内酯结构的螺环化 合物 63, 他们首次尝试了利用催化剂 C-16 与路易斯酸 共催化的催化体系来合成氧化吲哚螺环衍生物, 该反应 不仅具有较高的产率、较好的对映选择性, 更重要的是 该方法是少数几个成功实现 NHC 介导的高烯醇对滇红 酮羰基的环加成反应(Scheme 10). 2014 年, Yao 等 ${ }^{[32]}$ 也 采用氮杂卡宾催化剂 C-13, 以相同的滇红衍生物与 $\alpha, \beta-$ 不饱和酯 64 为原料, 实现了 $[4+2]$ 环加成反应, 该反应 最大的特点在于实现了 $\mathrm{NHC}$ 对 $\alpha, \beta$-不饱和酯的活化, 以较高的收率和立体选择性获得了目标产物 65 (Scheme 10). 另外, Glorius 等 ${ }^{[33]}$ 以 $\beta$ 位引入双取代基的 $\alpha, \beta$-不饱和醛 66 为原料, 实现与滇红衍生物的 $[3+2]$ 环 合反应, 获得了较高的收率和立体选择性. 通过研究, 他们发现该反应的高立体选择性主要取决于所加的路 易斯酸共催化剂(Scheme 10). 


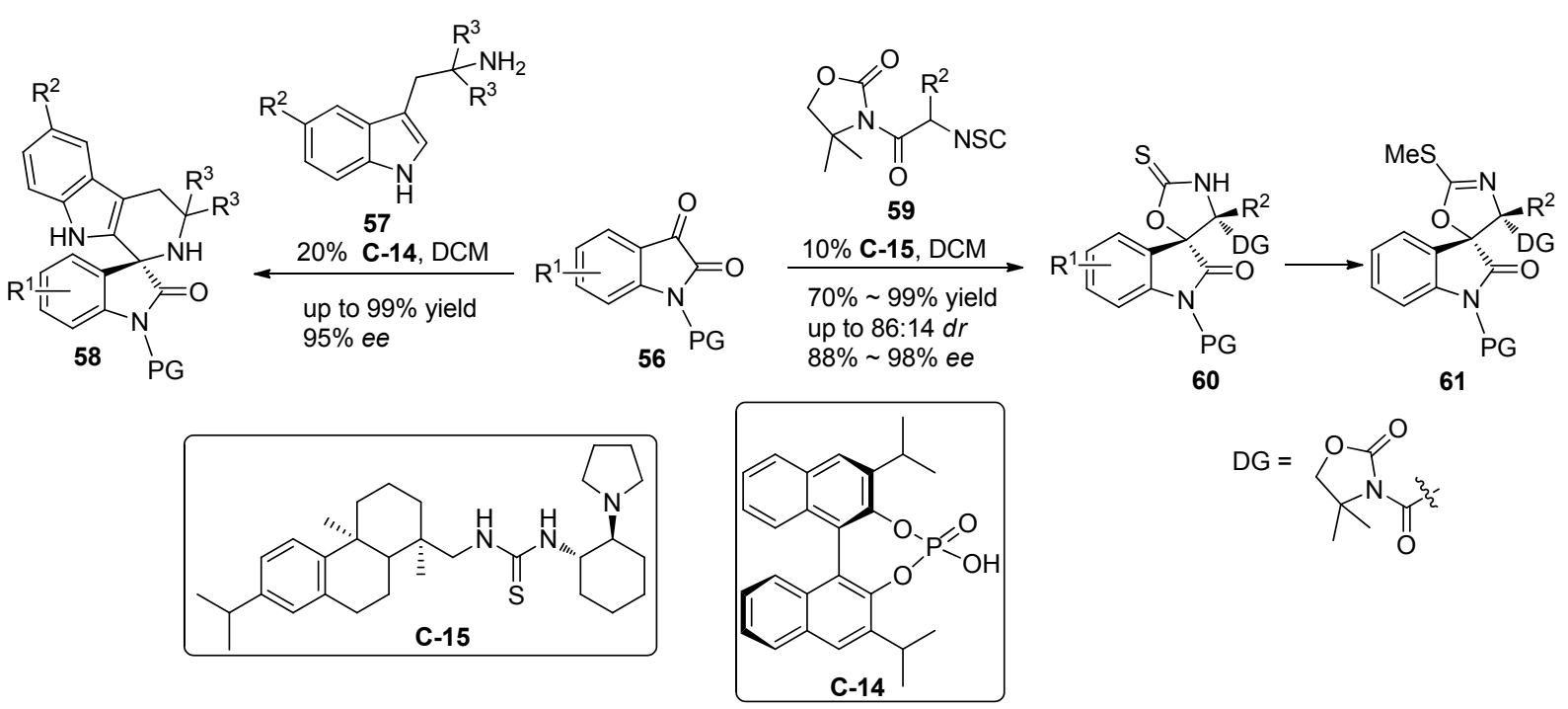

图式 $9 \mathrm{~N}$ 位保护的滇红为原料构建吲哚螺环骨架

Scheme 9 N-Protection isatin as starting materials to build spirooxindoles<smiles>[R]C=CC=O</smiles>

62<smiles>[R11]c1ccc2c(c1)C(=O)C(=[O+])N2[R2]</smiles>

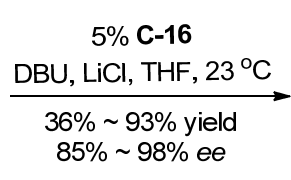

$\%$ 98\% ee
$10 \%$ C-17<smiles>[R1]C=C1C(=O)N([R])c2c[R1]ccc21</smiles><smiles>[R]C([R])=CC=O</smiles>

$\stackrel{\mathrm{THF}, 40^{\circ} \mathrm{C}, \mathrm{DBU}, t-\mathrm{BuCOOH},}{\longrightarrow}$

$68 \% \sim 98 \%$ yield $8: 1 \sim>20: 1 d r$

62

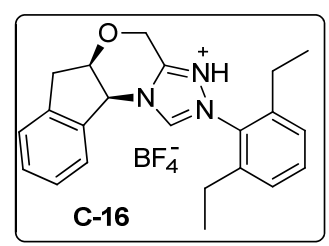

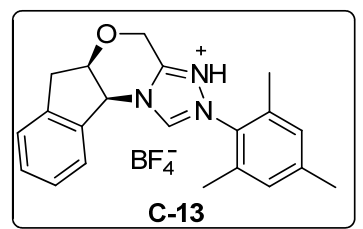

C-13<smiles>[R]C1CC(=O)O[C@@]12C(=O)N(C)C1=C[Z2]([H])C=CC=C12</smiles>

$63 R^{2}$

$\mathrm{R}^{1}$<smiles>[R][R]1cccc2c1N([Z7])C(=O)[C@]21CC([Y])=CC(=O)O1</smiles>

65

图式 $10 \mathrm{NHC}$ 介导的 $\alpha, \beta$-不饱和醛、 $\alpha, \beta$-不饱和酯与滇红的环加成反应构建吲哚螺环骨架

Scheme 10 NHC-catalyzed cycloaddition reaction of $\alpha, \beta$ unsaturated materias with isatin

\section{2 饱和氧化吲哚衍生物为起始原料}

\subsection{1 $\mathrm{C}(3)$ 位 $\mathrm{C}-\mathrm{C}$ 饱和氧化吲哚作为亲核试剂}

2012 年, 我们组 ${ }^{[34]}$ 在前期工作的基础上, 通过易得 的 2-氧化吲哚-3-羧酸酯(68)与硝基烯 69 反应, 一锅构建 具有五元内酰胺结构的氧化吲哚螺环化合物 $\mathbf{7 0}$, 该方 法具有原料简单的优点以及较好选择性, 其最大的意义 在于首次引入了双功能硫脲催化剂和布朗斯特酸来共
催化合成氧化吲哚螺环. 通过该方法可以构建多种结构 不同的氧化吲哚骨架, 且具有潜在的生物学活性 (Scheme 11). Barbas 等 ${ }^{[35]}$ 以 N-Boc 保护 3-取代氧化吲哚 衍生物 71 为原料, 在金鸡纳碱衍生物的催化下与类似 的硝基烯反应，通过一锅 Michael-Henry 级联反应高效 高选择性地获得多手性中心的吲哚螺环产物 72 (Scheme 11). 在 2015 年, Liu 等 ${ }^{[36]}$ 成功以钯和手性磷酸衍生物为 
催化剂, 探索了 $\mathrm{N}$ 无保护的吲哚衍生物 73 与乙烯基环丙 烷 74 之间的 $[3+2]$ 环加成反应, 其最大的特点在于该方 法首次实现了两性离子 $\pi$-烯丙基- $\mathrm{Pa}$-复合物中的碳负离 子与由反应过程中产生的亚胺之间的环加成反应. 该反 应使用了 $\mathrm{N}$ 无保护且反应活性较弱的吲哚为起始原料, 获得较好的收率和立体选择性(Scheme 11).

Me-DuPhos 是一类亲核磷化氢类催化剂, 具有活性 高、适用范围广的优点. Liu 等 ${ }^{[37]}$ 首次将 Me-DuPhos 催 化剂用于 $\mathrm{MBH}$ 碳酸盐 76 和 $N$-苯基马来酰亚胺(77)的 $[3+2]$ 环加成反应中, 实现了高效、高对映选择性和非 对映选择性地构建氧化吲哚螺环产物 78 (Scheme 12). 基于类似的反应底物, Chen 等 ${ }^{[38]}$ 采用 $\beta-6$ '-烷氧基异辛可 宁催化剂成功地实现了与炔烃 79 的不对称 $[3+2]$ 环加 成反应(Scheme 12).

\subsection{2 $\mathrm{C}(3)$ 位 $\mathrm{C}-\mathrm{N}$ 饱和氧化吲哚作为亲核试剂}

咪唑烷类吲哚螺环是一类重要的类药性骨架, 我们 课题组 ${ }^{[39]}$ 首次以 3-异硫氰酸酯氧化吲哚 82 为起始原料, 与苯基取代亚胺 83 在手性硫脲二胺类催化剂的作用下, 通过 Mannich/Cyclization 一锅串联快速构建此类吲哚螺 环骨架, 获得了良好的收率和对映选择性, 同时该类骨
架还可以进一步转化成具有潜在生物活性的 Spirobrassinin (Scheme 13). 基于类似的 3-异硫氰酸酯氧化吲哚 85, Wang 等 ${ }^{[40]}$ 采用 3-异硫氰酸酯氧化吲哚 85 为底物, 在非手性三乙胺的催化下, 成功实现了与 1,3-偶极偶氮 甲碱亚胺之间的 $[3+3]$ 环加成反应, 以高产率和非对映 选择性合成了 3,3'-三嗪基氧化吲哚螺环产物 87 (Scheme 13). 同样, Yuan 等 ${ }^{[41]}$ 引入 3-甲基- 4-硝基-5-烯基异啞唑 底物 90, 在手性奎宁的催化下以温和的反应条件实现 了 Michael/cyclization 串联反应, 得到了一系列具有三 个连续手性中心的螺环化合物 90 (Scheme 13).

\subsection{3 $\mathrm{C}(3)$ 位 $\mathrm{C}-\mathrm{O}$ 饱和氧化吲哚作为亲核试剂}

2011 年, Bergonzini 和 Melchiorre 课题组 ${ }^{[42]}$ 提出了 以羟基氧化吲哚 91 与具有双取代 $\alpha, \beta$-不饱和醛 92 为原 料合成吲哚螺丁内酯类化合物 93 的方法, 其最大的特 点是可以通过控制反应条件来选择性获得目标产物 (Scheme 14). 除了有机催化剂之外, 金属 $\mathrm{Ru}$ 络合物也 被用于手性吲哚螺环的合成. 2013 年, Krische 等 ${ }^{[43]}$ 以着弪 基氧化吲哚 94 为起始原料, 在 $\mathrm{Ru}_{3}(\mathrm{CO})_{12}$ 的作用下实现 类似的吲哚螺丁内酯类化合物 96 的构建, 具有良好的 收率(Scheme 14).<smiles>[R]C=C[N+](=O)[O-]</smiles><smiles>[R]C=C[N+](=O)[O-]</smiles><smiles>[R]C(C(=O)O)C1c2ccccc2NC1C</smiles>

73
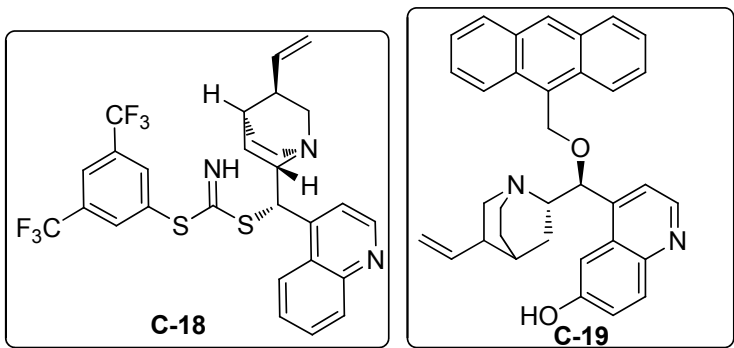

(1) $10 \% \mathrm{C}-18,10 \% \mathrm{PhCOOH}$ $\mathrm{CH}_{2} \mathrm{Cl}_{2},-70^{\circ} \mathrm{C}, 30 \mathrm{~h}$

(2) $\mathrm{Zn} / \mathrm{HOAc}, 70^{\circ} \mathrm{C}, 12 \mathrm{~h}$ $91 \% \sim 99 \%$ yield up to $92: 8 d r$ $86 \% \sim 97 \%$ ee

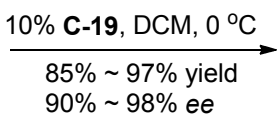

$5 \% \operatorname{Pd}(\mathrm{dba})_{2}, 15 \% \mathrm{C}-20$ $\mathrm{THF}, 10^{\circ} \mathrm{C}$

up to $74 \%$ yield up to $94 \%$ ee 74 $\mathrm{CO}_{2} \mathrm{Me}$ $\mathrm{CO}_{2} \mathrm{Me}$<smiles>[R2]C1CNC(=O)[C@@]12C(=O)N(C)c1ccccc12</smiles>

70<smiles>[R]C1[C@H]([N+](=O)[O-])C([R])[C@]2(CC1([R])O)c1ccccc1N2C(=O)OC(C)(C)C</smiles>

72

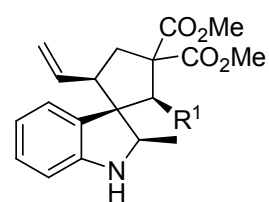

75

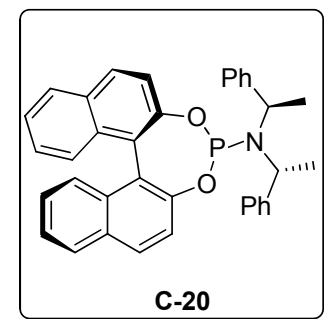

图式 $11 \mathrm{C}(3)$ 位单取代饱和氧化吲哚衍生物为起始原料构建吲哚螺环骨架

Scheme 11 Oxindole with one substitute on C(3) carbon as substrate to build spirooxindols 


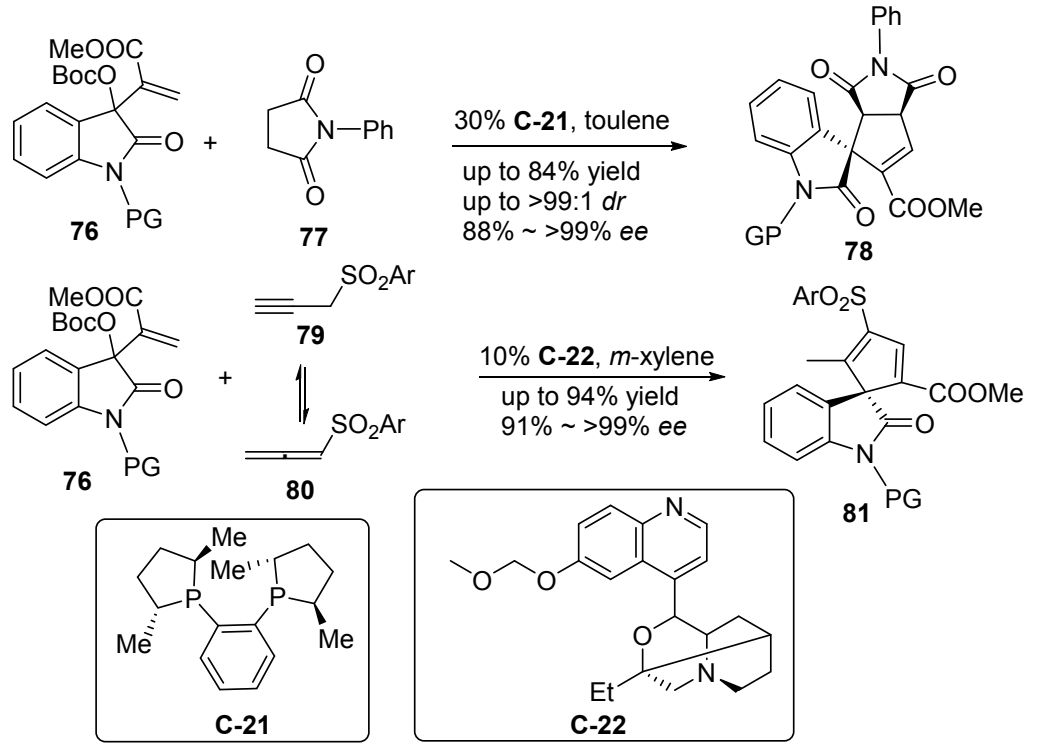

图式 $12 \mathrm{MBH}$ 碳酸盐和偶极子的 $[3+2]$ 环加成反应

Scheme $12[3+2]$ cycloaddition between $\mathrm{MBH}$ carbonates and dipolarophiles

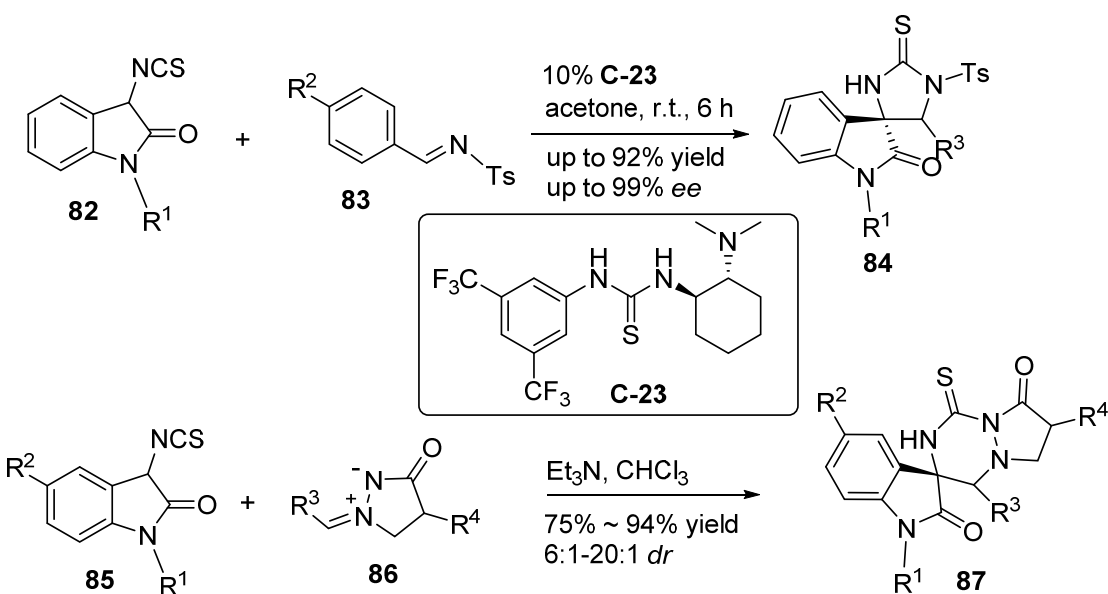<smiles>O=C1Nc2ccccc2C1[N+](=O)[O-]</smiles>

$88 R^{1}$<smiles>[3H]/C=C/c1onc(C)c1[N+](=O)[O-]</smiles>

89

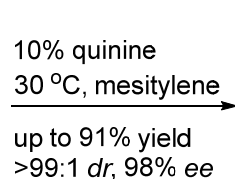
$>99: 1 d r, 98 \%$ ee

图式 13 3-异硫氧酸酯氧化吲哚为起始原料构建含硫吲哚螺环骨架

Scheme 13 Construction of spirooxindoles with sulfur by 3-isothiocyanateoxindol<smiles>O=C1Nc2ccccc2C1O</smiles>

91<smiles>[R]C([R])=CC=O</smiles>

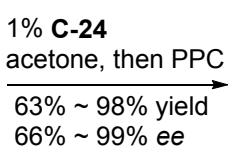
$66 \% \sim 99 \%$ ee<smiles>[R]C([R])=C([R])C(=O)OC</smiles>

$2 \% \mathrm{Ru}_{3}(\mathrm{CO})_{12}$ DPPP, $t$-BuOK $\underset{\text { up to } 94 \% \text { yield }}{\stackrel{m \text {-xylene, } 140^{\circ} \mathrm{C}, 20 \mathrm{~h}}{\longrightarrow}}$<smiles>[R7]C1CC(=O)OC12C(=O)Nc1ccccc12</smiles>

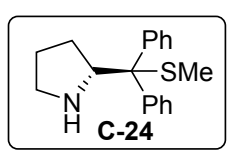

93<smiles>[R]C1C(=O)OC2(C(=O)N(Cc3ccccc3)c3ccccc32)C1([R])[R]</smiles>

图式 14 3-差基氧化吲哚构建吲哚螺环产物

Scheme 14 3-Hydroxyoxindol as starting materials to build spirooxindoles 


\section{$1.3 \mathrm{C}(3)$ 位无取代的氧化吲哚衍生物为起始原料}

$C(3)$ 位无取代的氧化吲哚由于可能具有两次亲核中 心, 使得该类反应变得更为复杂. Rios 等 ${ }^{[44]}$ 巧妙地利用无 取代氧化哚具有两次亲核中心的特点, 与 $\alpha, \beta$-不饱和 醛 3 在吡咯烷催化剂 C-1 的作用下, 一锅实现了两次亲核 加成的串联反应, 高效高立体选择性生成了目标螺环产 物 98 (Scheme 15). 2012 年, $\mathrm{Lu}$ 等 ${ }^{[45]}$ 以 C-25 为催化剂, 实 现了氧化吲哚 99 与酩 100 加成反应, 构建了一类多样化 的氧化吲哚螺环内酯化合物 101 (Scheme 15). 同样, Xu 等 ${ }^{[46]}$ 以手性硫脲催化剂 C-26 一锅实现了无取代氧化吲 哚 102 烯酮酯 $103[5+1]$ 环加成反应, 以高对映选择性和 非对映选择性得到了具有 $\delta$-内酯结构的吲哚螺环骨架
104. 他们研究发现氧化吲哚 $\mathrm{N}$ 位不同的取代基对反应的 活性以及选择性有着不同的影响(Scheme 15).

近年来，同一反应中多个催化剂协同催化的模式引 起了人们越来越多的关注. Zhou 等 ${ }^{[47]}$ 在构建多手性中 心的吲哚螺环骨架的研究中, 采用了吡咯烷类催化剂 C-1 和奎尼丁硫嫝类双催化剂 C-27, 有效地实现了 C(3) 无取代的 N-Boc 保护的氧化吲哚 105 分别与硝基烯 69 和 $\alpha, \beta$-不饱和醛 3 一锅串联反应, 以较高的收率和较好 的立体选择性生成了吲哚螺环己烷类化合物 106. 同时, 他们的研究发现, 催化剂 C-28 中 TBS 取代基的引入对 产物的构型有着重要的影响, 获得构型反转的目标化合 物 107 (Scheme 16).

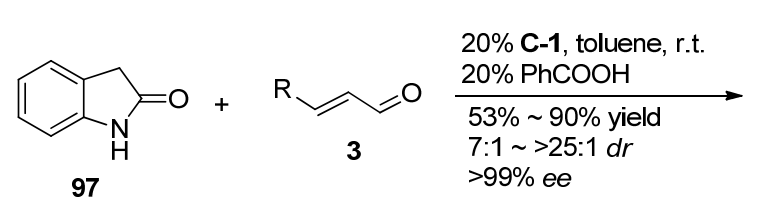<smiles>[R][R]C#CC=O</smiles>

$30 \%$ C-25, 30\% $t$-BuOK THF, $65^{\circ} \mathrm{C}, 2 \mathrm{~h}, 4 \AA \mathrm{AS}$ up to $93 \%$ yield up to $>95: 5 d r$

99<smiles>[R]C1CC=C(C=O)C([R])C12C(=O)Nc1ccccc12</smiles><smiles>[R]C#C[C@H]1OC(=O)C[C@@H]([R])[C@]12C(=O)N([R2])c1cc[R1]([H])cc12</smiles>

101

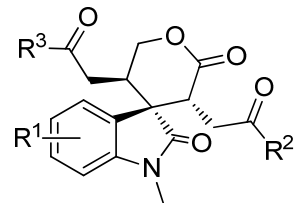<smiles>[R1]c1ccc2c(c1)CC(=O)N2Cc1cc(NC(=S)N[C@H](CN2CCCCC2)C(C)(C)C)cc(C(F)(F)F)c1</smiles>
$\frac{10 \% \text { C-26, toluene, r.t. }}{\text { uo to } 94 \% \text { yield }}$
$97 \%$ ee

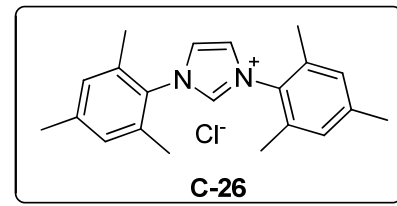

104

图式 15 无取代氧化吲哚底物构建吲哚螺环骨架

Scheme 15 Use of nonsubstituted oxindole as reactants<smiles>[R]C1CC(O)C2(C(=O)N(C(=O)OCc3ccccc3)c3ccccc32)C([R])C1[N+](=O)[O-]</smiles>

up to $92 \%$ yield 9:2.5:1 $d r,>99 \%$ ee

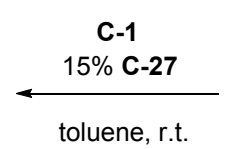

toluene, r.t.

$$
\text { (n) }
$$<smiles>CC(C)(C)OC(=O)N1C(=O)Cc2ccccc21</smiles>

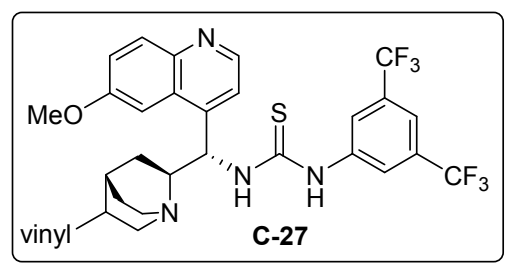<smiles>[R]C=C[N+](=O)[O-]</smiles>

C-28 69 0 3 $15 \%$ C-27 oluene, r.t.

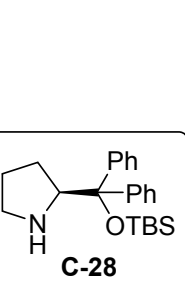<smiles>[R]C1CC(O)C2(C(=O)N(C(=O)OCc3ccccc3)c3ccccc32)C([R2])C1[N+](=O)[O-]</smiles>

up to $94 \%$ yield 7:2:1 dr, >99\% ee

图式 16 协同催化剂催化 N-Boc 保护的氧化吲哚分别与硝基烯和 $\alpha, \beta$-不饱和醛的 Michael/Michael/Aldol 环加成反应 Scheme 16 Relay Michael/Michael/aldol addition reactionof N-substituted oxindoles, nitrostyrenes and $\alpha, \beta$-unsaturated aldehydes by synergistic catalysis 
2012 年, Dou 和 $\mathrm{Lu}$ 课题组 ${ }^{[48]}$ 首先提出是否可以将 $\mathrm{C}(3)$ 位无取代的氧化吲哚衍生物与卤代硝基烯混合来 手性合成 C(3)位具有环丙烷取代的氧化吲哚螺环产物. 通过篎选不同的催化剂, 他们最终发现催化剂 C-29 可 以以较高的收率、较好的对映选择性与非对映选择性得 到目标产物 110, 且在该反应体系中需要加入化学计量 的 $\left(\mathrm{NH}_{4}\right)_{2} \mathrm{CO}_{3}$ 来消耗反应过程中产生的 $\mathrm{HBr}$. 通过后期 的研究, 他们发现当向反应体系加入催化量的亲核催化 剂 DABCO 时, 则会生成构型反转的产物 111 (Scheme 17).

\section{4 其他化合物为起始原料}

吲哚螺环骨架基本都是通过氧化吲哚衍生物与其 他小分子底物的串联反应来实现. 但是，近年来随着合 成化学的不断发展, 化学家也成功实现了以非氧化吲哚 化合物为底物来构建复杂吲哚螺环骨架. 2011 年, Gong 等 ${ }^{[49]}$ 首次利用三个简单的原料在催化剂 C-30 的作用下 全合成出具有抗 tsFT210 细胞扩散功能的氧化吲哚螺环 化合物 115 的重要中间体 114, 该方法因其较好的收率 和立体选择性而受到广泛关注，该中间体可以很容易地 转化为化合物 115 (Scheme 18). 通常情况下，氧化吲哚<smiles></smiles>

110

up to $81 \%$ yield $>20: 1 d r, 99 \%$ ee

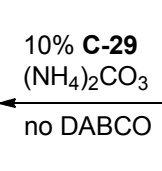<smiles>O=C1Cc2c[R11]ccc2N1</smiles>

108 Boc

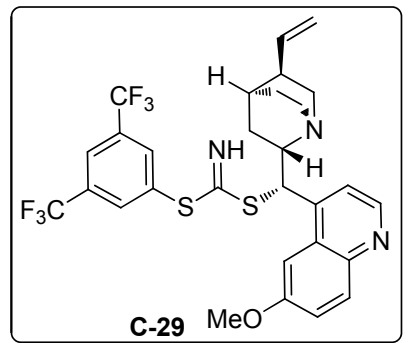<smiles>[R]C=C(Br)[N+](=O)[O-]</smiles>

109

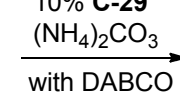<smiles>[R]C1[C@H]([N+](=O)[O-])[C@@]12C(=O)N(C(C)(C)C)c1cc[R1]cc12</smiles>

up to $95 \%$ yield $>20: 1 d r, 98 \%$ ee

$\mathrm{DABCO}=1,4-$ diazabicyclo[2.2.2]octane

图式 17 无取代氧化吲哚与卤代硝基烯的级联反应生成吲哚螺环丙烷产物

Scheme 17 Cascade reaction of oxindoles with halo-nitroolefins to give spirocyclopropyl oxindoles

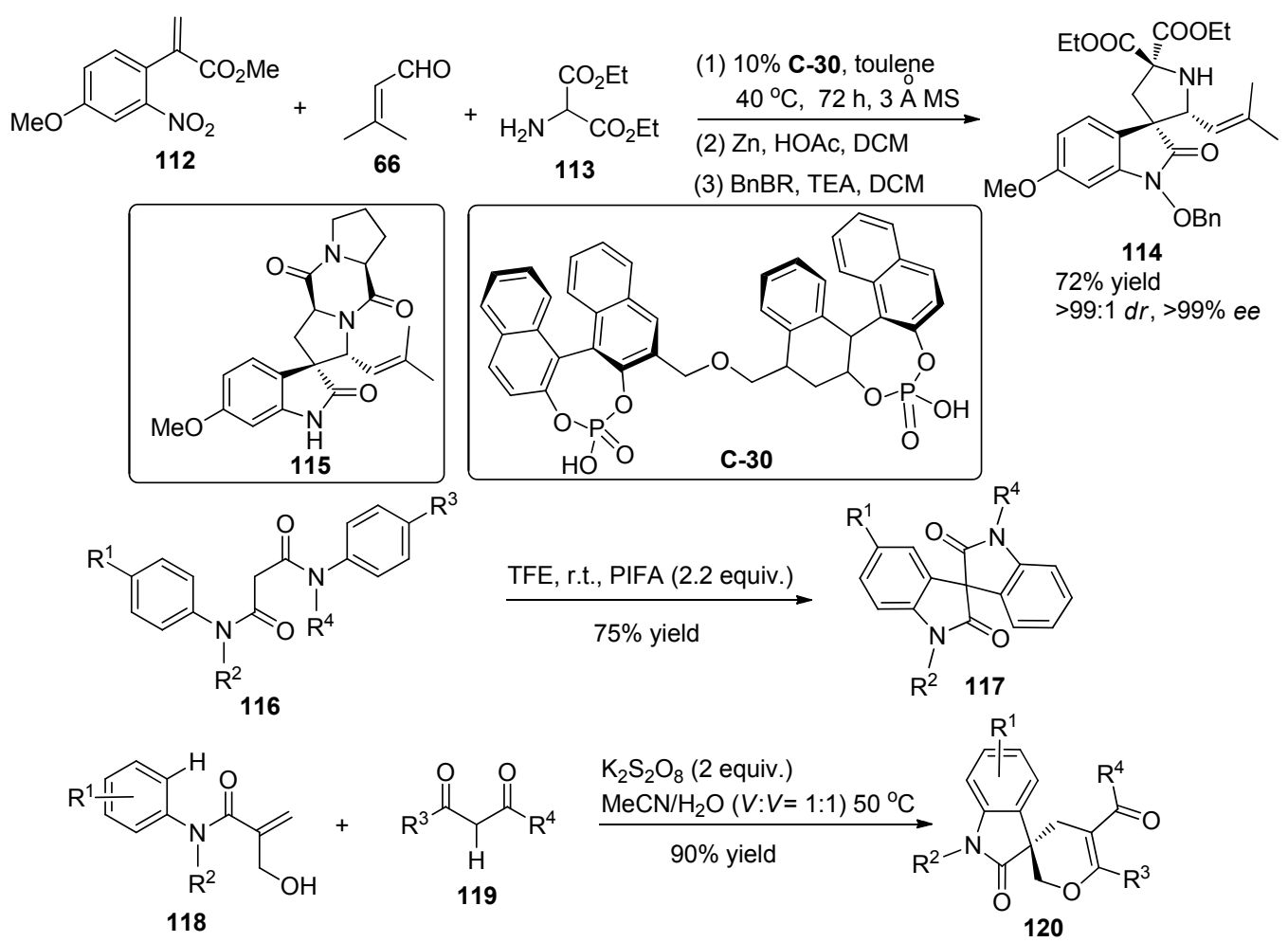

图式 18 非氧化吲哚底物构建吲哚螺环骨架

Scheme 18 Nonoxindoles as reactants to build spiroindoles 
螺环化合物都是由两种或两种以上起始原料通过级联 反应合成的, 2012 年, Zhao 等 ${ }^{[50]}$ 首次报道了利用 PIFA 为催化剂, 通过单一起始原料 116 发生分子内的环合反 应来构建具有 $\gamma$-内酰胺结构的吲哚螺环 117 的新方法 (Scheme 18), 该方法具有操作简便、产率适中等优点. 该方法的另一个重要意义在于他们实现了非金属氧化 的 $\mathrm{C}\left(\mathrm{sp}^{2}\right)-\mathrm{C}\left(\mathrm{sp}^{3}\right)$ 键的形成. 2013 年, Duan 等 ${ }^{[51]}$ 报道了一 个以羟基取代 $\alpha, \beta$-不饱和酰胺 118 与 1,3-二羰基化合物 119 为原料构建具有环己醚结构的吲哚螺环 120 的新方 法, 该反应过程包含两个新的 $\mathrm{C}-\mathrm{C}$ 键以及一个 $\mathrm{C}-\mathrm{O}$ 键形成, 且反应条件温和, 产率较高(Scheme 18).

2014 年, Eycken 等 ${ }^{[52]}$ 通过共催化的策略成功合成 吲哚螺环 $\gamma$-内酰胺结构化合物 $\mathbf{1 2 5}$, 该反应是以醋酸钯 与 Xantphos 为共催化剂, 实现了 Buchwald-Hartwig/ Michael 串联反应，具有原料简单易得、反应快速和产率 适中的优点(Scheme 19). 另外, Maison 等 ${ }^{[53]}$ 也报道了一 个在钯催化下合成功能性四氢吡咯吲哚螺环 128 的反 应，该反应可以利用较简单的原料以较少的步骤合成天 然产物及其衍生物, 并且反应具有较高的产率(Scheme 19).

\section{2 结果与展望}

氧化吲哚螺环化合物多含有电负强的氧和氮等杂 原子, 可以与其它生物大分子分之间有较大的分子间作
用力, 因此是一类重要的类药性骨架. 近年来, 国内外 学者越来越重视该领域的研究，合成了成千上万种具有 氧化吲哚螺环骨架的化合物, 期望可以从中笁选出了一 批有药效的化合物. 许多人工合成的螺环化合物(包括 天然提取物中得到的螺环化合物)一般都是消旋化合物, 而其中的 $R$ 构型和 $S$ 构型的药理和毒理是不一样的. 但 是从天然产物中分离出来或是通过传统方法化学合成 的吲哚螺环产物几乎都是消旋体，或者立体选择性不 高. 因此，寻找高效、高选择性构建吲哚螺环骨架的方 法已成为有机化学家和药物化学家日益关注的问题.

通过手性有机催化剂介导的有机串联反应策略不 对称合成氧化吲哚螺环类化合物由于其产率和立体选 择性高已成为近年来的研究热点. 在过去的几年中, 我 们见证了有机催化剂的快速发展. 由于其催化的高效 性、立体选择的专一性而得到了有机化学家的亲睐, 最 终被应用于构建各种各样的氧化吲哚螺环骨架. 但是由 于有机催化剂存在种类少、一种催化剂所能催化的反应 种类单一以及构建吲哚螺环的底物范围窄等局限, 发展 一类高效、高选择性且适用范围广的有机催化剂势必成 为我们今后研究的重点. 另外, 由于 $\mathrm{NHC}$ 类催化剂独 特的性质, 也将在吲哚螺环的构建中发挥越来越重要的 作用. 我们相信一些以氧化吲哚螺环骨架为基础的新化 合物，将被广泛地用于药物化学的研究中.
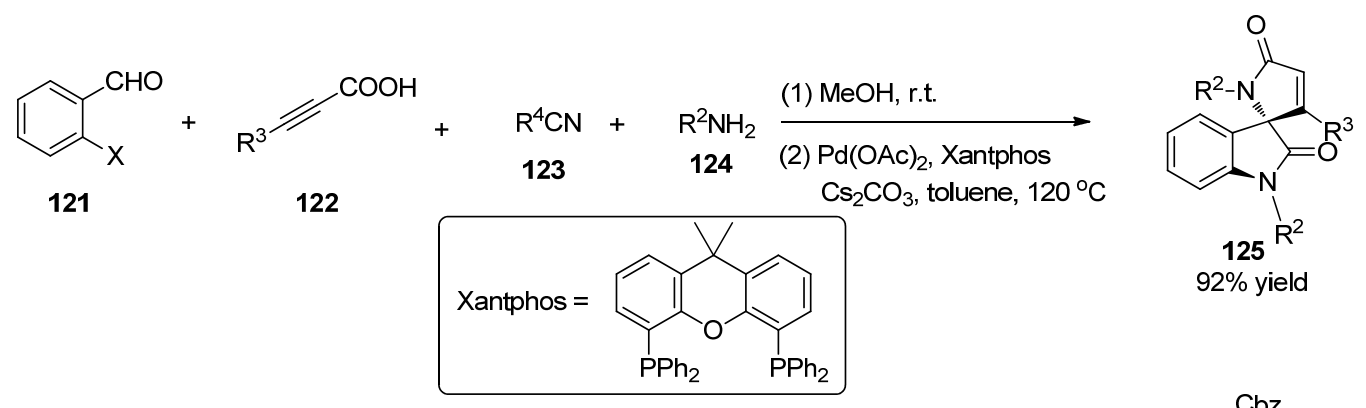

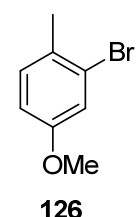

126

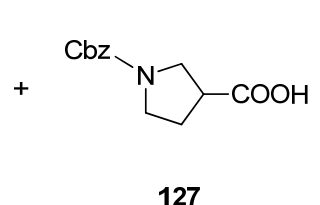

127
(1) $\mathrm{PCl}_{5}$, DIEA, $0^{\circ} \mathrm{C}$

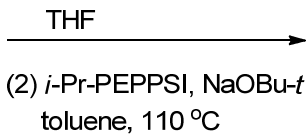
toluene, $110^{\circ} \mathrm{C}$

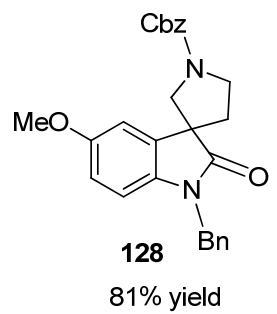

图式 19 钯催化非氧化吲哚底物构建吲哚螺环

Scheme 19 Synthesis of siproindoles catalyzed by palladium

\section{References}

[1] Lin, H.; Danishefsky, S. J. Angew. Chem., Int. Ed. 2003, 42, 36.

[2] Ding, K.; Lu, Y.; Nikolovska-Coleska, Z.; Qiu, S.; Ding, Y.; Gao, W.; Stuckey, J.; Krajewski, K.; Roller, P. P.; Tomita, Y.; Parrish, D. A.; Deschamps, J. R.; Wang, S. J. Am. Chem. Soc. 2005, 127, 10130 .
[3] Shangary, S.; Qin, D.; McEachern, D.; Liu, M.; Miller, R. S.; Qiu, S.; Nikolovska-Coleska, Z.; Ding, K.; Wang, G.; Chen, J.; Bernard, D.; Zhang, J.; Lu, Y.; Gu, Q.; Shah, R. B.; Pienta, K. J.; Ling, X.; Kang, S.; Guo, M.; Sun, Y.; Yang, D.; Wang, S. Proc. Natl. Acad. Sci. U. S. A. 2008, 105, 3933.

[4] (a) Rottmann, M.; McNamara, C.; Yeung, B. K.; Lee, M. C.; Zou, B.; Russell, B.; Seitz, P.; Plouffe, D. M.; Dharia, N. V.; Tan, J.; 
Cohen, S. B.; Spencer, K. R.; Gonzalez-Paez, G. E.; Lakshminarayana, S. B.; Goh, A.; Suwanarusk, R.; Jegla, T.; Schmitt, E. K.; Beck, H. P.; Brun, R.; Nosten, F.; Renia, L.; Dartois, V.; Keller, T. H.; Fidock, D. A.; Winzeler, E. A.; Diagana, T. T. Science. 2010, $329,1175$.

(b) Yeung, B. K.; Zou, B.; Rottmann, M.; Lakshminarayana, S. B.; Ang, S. H.; Leong, S. Y.; Tan, J.; Wong, J.; Keller-Maerki, S.; Fischli, C.; Goh, A.; Schmitt, E. K.; Krastel, P.; Francotte, E.; Kuhen, K.; Plouffe, D.; Henson, K.; Wagner, T.; Winzeler, E. A.; Petersen, F.; Brun, R.; Dartois, V.; Diagana, T. T.; Keller, T. H. J. Med. Chem. 2010, 53, 5155.

[5] Jiang, K.; Jia, Z. J.; Yin, X.; Wu, L.; Chen, Y. C. Org. Lett. 2010, $12,2766$.

[6] (a) Erkkila, A.; Majander, I.; Pihko, P. M. Chem. Rev. 2007, 107, 5416.

(b) Melchiorre, P. Angew. Chem., Int. Ed. 2012, 51, 9748.

(c) Mukherjee, S.; Yang, J. W.; Hoffmann, S.; List, B. Chem. Rev. 2007, 107, 5471.

(d) Xu, L. W.; Luo, J.; Lu, Y. Chem. Commun. 2009, 1807.

[7] Bencivenni, G.; Wu, L. Y.; Mazzanti, A.; Giannichi, B.; Pesciaioli, F.; Song, M. P.; Bartoli, G.; Melchiorre, P. Angew. Chem., Int. Ed. 2009, 48, 7200 .

[8] Jiang, K.; Jia, Z. J.; Chen, S.; Wu, L.; Chen, Y. C. Chemistry 2010, $16,2852$.

[9] Liu, Y.; Nappi, M.; Arceo, E.; Vera, S.; Melchioree, P. J. Am. Chem. Soc. 2011, 133, 15212.

[10] Wang, G.; Liu, X.; Huang, T.; Kuang, Y.; Lin, L.; Feng, X. Org. Lett. 2013, 15, 76.

[11] Qi, L. W.; Yang, Y.; Gui, Y. Y.; Zhang, Y.; Chen, F.; Tian, F.; Peng, L.; Wang, L. X. Org. Lett. 2014, 16, 6436.

[12] Doyle, A. G.; Jacobsen, E. N. Chem. Rev. 2007, 107, 5713.

[13] Zhang, Z.; Schreiner, P. R. Chem. Soc. Rev. 2009, 38, 1187.

[14] Tan, B.; Hernandez-Torres, G.; Barbas, C. F. J. Am. Chem. Soc. 2011, 133, 12354.

[15] Mao, H.; Lin, A.; Tang, Y.; Shi, Y.; Hu, H.; Cheng, Y.; Zhu, C. Org. Lett. 2013, 15, 4062.

[16] Tan, B.; Candeias, N. R.; Barbas, C. F. J. Am. Chem. Soc. 2011, $133,4672$.

[17] Zhu, G.; Sun, W.; Wu, C.; Li, G.; Hong, L.; Wang, R. Org. Lett. 2013, 15, 4988.

[18] Zhao, Q. Y.; Lian, Z.; Wei, Y.; Shi, M. Chem. Commun. 2012, 48, 1724.

[19] Voituriez, A.; Pinto, N.; Neel, M.; Retailleau, P.; Marinetti, A. Chemistry 2010, 16, 12541.

[20] Chen, X. H.; Wei, Q.; Luo, S. W.; Xiao, H.; Gong, L. Z. J. Am. Chem. Soc. 2009, 131, 13819.

[21] Li, G.; Liang, T.; Wojtas, L.; Antilla, J. C. Angew. Chem., Int. Ed. 2013, 52, 4628.

[22] Zhong, F.; Han, X.; Wang, Y.; Lu, Y. Angew. Chem., Int. Ed. 2011, 50,7837 .

[23] Zhong, F.; Han, X.; Wang, Y.; Lu, Y. Chem. Sci. 2012, 3, 1231.

[24] Suman, K.; Srinu, L.; Thennarasu, S. Org. Lett. 2014, 16, 3732.

[25] Badillo, J. J.; Ribeiro, C. J.; Olmstead, M. M.; Franz, A. K. Org.
Lett. 2014, 16, 6270

[26] Chen, X.; Chen, H.; Ji, X.; Jiang, H.; Yao, Z. J.; Liu, H. Org. Lett. 2013, 15, 1846.

[27] Xu, J.; Mou, C.; Zhu, T.; Song, B. A.; Chi, Y. R. Org. Lett. 2014, $16,3272$.

[28] Duce, S. P., F.; Gramigna, L.; Bernardi, L.; Mazzanti, A.; Ricci, A.; Bartoli, G.; Bencivenni, G. Adv. Synth. Catal. 2011, 353, 5.

[29] Badillo, J. J.; Silva-Garcia, A.; Shupe, B. H.; Fettinger, J. C.; Franz, A. K. Tetrahedron Lett. 2011, 52, 5550.

[30] Jiang, X.; Cao, Y.; Wang, Y.; Liu, L.; Shen, F.; Wang, R. J. Am. Chem. Soc. 2010, 132, 15328.

[31] Dugal-Tessier, J.; O'Bryan, E. A.; Schroeder, T. B.; Cohen, D. T.; Scheidt, K. A. Angew. Chem., Int. Ed. 2012, 51, 4963.

[32] Que, Y.; Li, T.; Yu, C.; Wang, X. S.; Yao, C. J. Org. Chem. 2015, 80, 3289.

[33] Li, J. L.; Sahoo, B.; Daniliuc, C. G.; Glorius, F. Angew. Chem., Int. Ed. 2014, 53, 10515.

[34] Chen, X. J.; Qian, W. K.; Feng, E. G.; Zhou, Y.; Wang, J. F.; Jiang, H. L.; Yao, Z. J.; Liu. H. Adv. Synth. Catal. 2012, 354, 6.

[35] Albertshofer, K.; Tan, B.; Barbas, C. F. Org. Lett. 2012, 14, 1834.

[36] Liu, Z. S.; Li, W. K.; Kang, T. R.; He, L.; Liu, Q. Z. Org. Lett. 2015, 17, 150.

[37] Wang, Y.; Liu, L.; Zhang, T.; Zhong, N. J.; Wang, D.; Chen, Y. J. J. Org. Chem. 2012, 77, 4143.

[38] Peng, J.; Huang, X.; Jiang, L.; Cui, H. L.; Chen, Y. C. Org. Lett. 2011, 13, 4584.

[39] Cai, H.; Zhou, Y.; Zhang, D.; Xu, J. Y.; Liu, H. Chem. Commun. 2014, 50, 14771.

[40] Sun, W.; Zhu, G.; Wu, C.; Li, G.; Hong, L.; Wang, R. Angew. Chem., Int. Ed. 2013, 52, 8633.

[41] Liu, X. L.; Han, W. Y.; Zhang, X. M.; Yuan, W. C. Org. Lett. 2013, $15,1246$.

[42] Bergonzini, G.; Melchiorre, P. Angew. Chem., Int. Ed. 2012, 51, 971.

[43] McInturff, E. L.; Mowat, J.; Waldeck, A. R.; Krische, M. J. J. Am. Chem. Soc. 2013, 135, 17230.

[44] Companyo, X.; Zea, A.; Alba, A. N.; Mazzanti, A.; Moyano, A.; Rios, R. Chem. Commun. 2010, 46, 6953.

[45] Du, D.; Hu, Z.; Jin, J.; Lu, Y.; Tang, W.; Wang, B.; Lu, T. Org. Lett. 2012, 14, 1274.

[46] Zhao, S.; Lin, J. B.; Zhao, Y. Y.; Liang, Y. M.; Xu, P. F. Org. Lett. 2014, 16, 1802.

[47] Zhou, B.; Yang, Y.; Shi, J.; Luo, Z.; Li, Y. J. Org. Chem. 2013, 78, 2897.

[48] Dou, X.; Lu, Y. Chem.-Eur. J. 2012, 18, 8315.

[49] Cheng, M. N.; Wang, H.; Gong, L. Z. Org. Lett. 2011, 13, 2418.

[50] Wang, J.; Yuan, Y.; Xiong, R.; Zhang-Negrerie, D.; Du, Y.; Zhao, K. Org. Lett. 2012, 14, 2210.

[51] Wang, H.; Guo, L. N.; Duan, X. H. Org. Lett. 2013, 15, 5254.

[52] Sharma, N.; Li, Z.; Sharma, U. K.; Van der Eycken, E. V. Org. Lett. 2014, 16, 3884.

[53] Deppermann, N.; Thomanek, H.; Prenzel, A.; Maison, W. J. Org. Chem. 2010, 75, 5994 . 